\title{
An Extension of the Generalized Linear Failure Rate Distribution
}

\author{
M. R. Kazemi*, A. A. Jafari ${ }^{\dagger}$, S. Tahmasebi ${ }^{\ddagger}$ \\ * Department of Statistics, Fasa University, Fasa, Iran \\ †Department of Statistics, Yazd University, Yazd, Iran \\ ${ }_{\ddagger}^{\ddagger}$ Department of Statistics, Persian Gulf University, Bushehr, Iran
}

\begin{abstract}
In this paper, we introduce a new extension of the generalized linear failure rate distributions. It includes some well-known lifetime distributions such as extension of generalized exponential and generalized linear failure rate distributions as special sub-models. In addition, it can have a constant, decreasing, increasing, upside-down bathtub (unimodal), and bathtub-shaped hazard rate function depending on its parameters. We provide some of its statistical properties such as moments, quantiles, skewness, kurtosis, hazard rate function, and reversible hazard rate function. The maximum likelihood estimation of the parameters is also discussed. At the end, a real data set is given to illustrate the usefulness of this new distribution in analyzing lifetime data.
\end{abstract}

Keywords: Generalized exponential distribution; Hazard function; Maximum likelihood estimation.

\section{Introduction}

The generalized linear failure rate (GLFR) distribution is defined by Sarhan and Kundu (2009) and contains various well-known distributions: the generalized exponential (GE) distribution introduced by Gupta and Kundu (1999), the generalized Rayleigh distribution introduced by Surles and Padgett (2001, 2005), the exponential, Rayleigh, and linear failure rate (LFR) distributions are its special cases. The GLFR distribution has decreasing or unimodal probability density function (pdf) and its hazard rate function (hrf) can have increasing, decreasing, and bathtub-shaped. Unfortunately, the GLFR distribution cannot have unimodal hrf.

*Corresponding: kazemi@fasau.ac.ir 
Recently, many studies have been done on GLFR distribution, and some authors have extended it: the generalized linear exponential (Mahmoud and Alam, 2010), beta-linear failure rate (Jafari and Mahmoudi, 2015), Kumaraswamy-GLFR (Elbatal, 2013), modified-GLFR (Jamkhaneh, 2014), McDonald-GLFR (Elbatal et al., 2014), Poisson-GLFR (Cordeiro et al., 2015), GLFR-geometric (Nadarajah et al., 2014), and GLFR-power series (Alamatsaz and Shams, 2014) are some univariate extension of GLFR distribution.

Kundu and Gupta (2011) proposed an extension of GE distribution that is a very flexible family of distribution. It is positively skewed, and has increasing, decreasing, unimodal and bathtub shaped hrfs. It is included GE, exponential, generalized Pareto (Johnson et al., 1995), and Pareto distributions.

Cordeiro et al. (2012) introduced a five-parameter called the McDonald extended exponential distribution as a generalization of extended generalized exponential (EGE). In this paper, we introduce a new four-parameter distribution that contains the EGE distribution as its special case. In addition, this class of distribution extends the three-parameter GLFR distribution. Therefore, it is called extended generalized linear failure rate (EGLFR) distribution. The hrf of this new distribution is increasing, decreasing, bathtub, and unimodal. In addition, we will show that the new distribution has been fit better than the EGE distribution and other competing distributions to analyzing the lifetime data.

The paper is organized as follows. In Section 2, we introduce a new class of distributions. Some statistical properties such as moments, quantiles, hrf, and reversible hazard rate are provided in Section 3. The maximum likelihood estimation (MLE) of the parameters is obtained in Section 4, An application of the EGLFR distribution using a real data set is presented in Section 5 .

\section{A new class}

In this section, we introduce a new class of distributions as extension of GLFR distribution. Also, some properties of the pdf and hrf of this distribution are given here.

Theorem 2.1. For given $\alpha>0, \beta \in \mathbb{R}, a \geq 0$, and $b \geq 0$ (with $a+b>0$ ), consider the function

$$
F(x)=\left\{\begin{array}{lll}
\left(1-\left(1-\beta\left(a x+\frac{b}{2} x^{2}\right)\right)^{1 / \beta}\right)^{\alpha} & \text { if } & \beta \neq 0 \\
\left(1-\mathrm{e}^{-\left(a x+\frac{b}{2} x^{2}\right)}\right)^{\alpha} & \text { if } & \beta=0
\end{array}\right.
$$

i. If $\beta \leq 0$, then $F(x)$ is a cumulative distribution function (cdf) on $(0, \infty)$. 
ii. If $\beta>0$ and $b \neq 0$, then $F(x)$ is a cdf on $(0, \psi)$ where $\psi=\frac{1}{b} \sqrt{a^{2}+\frac{2 b}{\beta}}-\frac{a}{b}$.

iii. If $\beta>0$ and $b=0$, then $F(x)$ is a cdf on $(0, \psi)$ where $\psi=\frac{1}{a \beta}$.

Proof. When $\beta=0$, proof is obvious. We consider $\beta \neq 0$. Without loss of generality, we consider $\alpha=1$. Therefore, $\lim _{x \rightarrow 0} F(x)=0$ and $F^{\prime}(x)=(a+b x)\left(1-\beta\left(a x+\frac{b}{2} x^{2}\right)\right)^{\frac{1}{\beta}-1}$.

i. When $\beta<0, \lim _{x \rightarrow \infty} F(x)=1$ and $F^{\prime}(x)>0$ for $0<x<\infty$.

ii. When $\beta>0, \lim _{x \rightarrow \psi} F(x)=1$ and $F^{\prime}(x)>0$ for $0<x<\psi$.

iii. This part is similar to part ii.

Therefore, proof is completed.

When the function $F$ in (2.1) is a cdf, it is said EGLFR distribution with parameters $\alpha, \beta$, $a$ and $b$, and will be denoted by $\operatorname{EGLFR}(\alpha, \beta, a, b)$. The pdf of this new class of distributions is

$$
f(x ; \alpha, \beta, a, b)=\left\{\begin{array}{lll}
\alpha(a+b x)(1-\beta z)^{\frac{1}{\beta}-1}\left(1-(1-\beta z)^{1 / \beta}\right)^{\alpha-1} & \text { if } & \beta \neq 0 \\
\alpha(a+b x) \mathrm{e}^{-z}\left(1-\mathrm{e}^{-z}\right)^{\alpha-1} & \text { if } & \beta=0
\end{array}\right.
$$

where $z=a x+\frac{b}{2} x^{2}$. The plots for pdf of EGLFR distribution are given in Figure 1, for some different values of parameters.
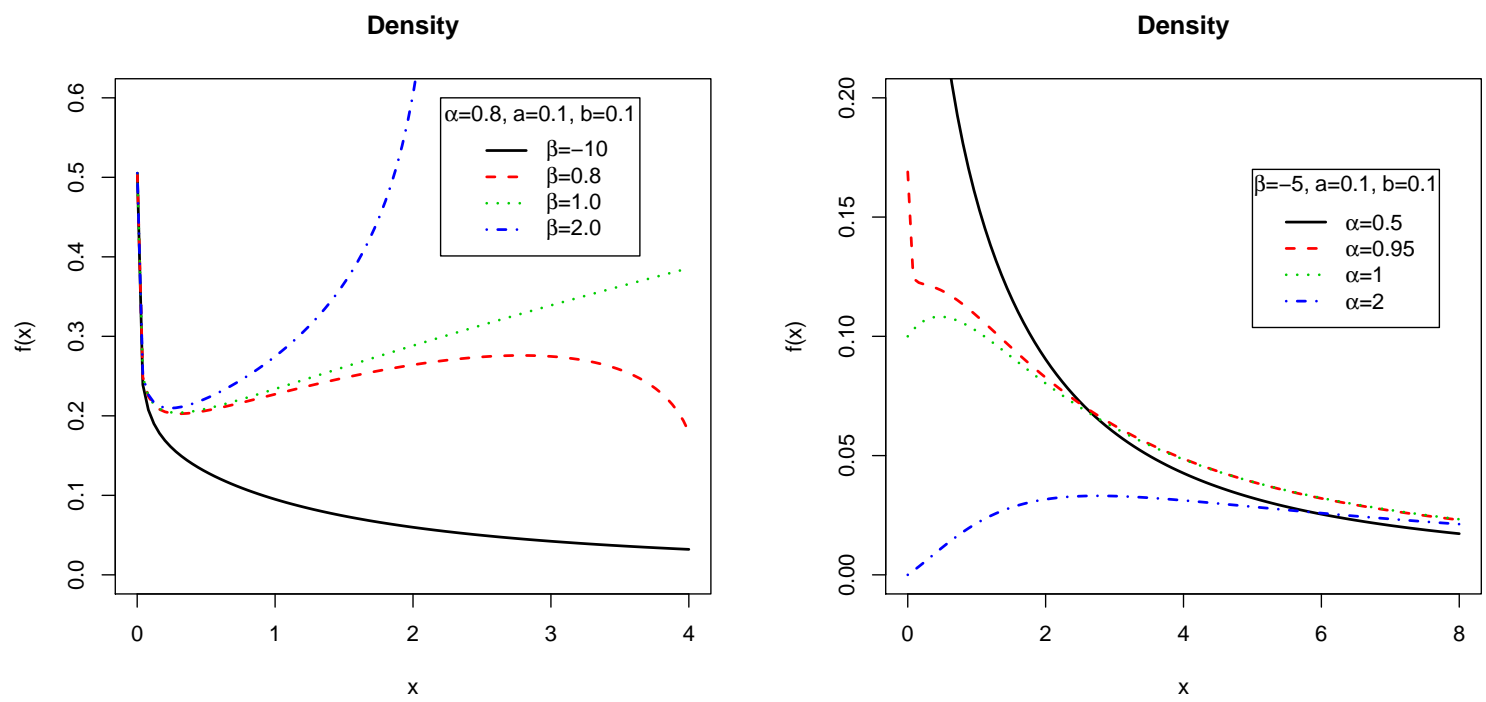

Figure 1: The plot of pdf for some different values of parameters.

If $\beta=0$, then this distribution reduces to the GLFR distribution which is introduced by Sarhan and Kundu (2009). The GLFR includes the GE (if $b=0$ ), exponential (if $b=0, \alpha=1$ ), LFR (if $\alpha=1$ ), generalized Rayleigh (if $a=0$ ) and Rayleigh (if $a=0, \alpha=1$ ) distributions as its special sub-models.

If $b=0$ then EGLFR distribution reduces to the EGE distribution introduced by Kundu and Gupta 
(2011). The EGE distribution includes GE (if $\beta=0$ ), exponential (if $\beta=0, \alpha=1$ ), generalized Pareto (if $\alpha=1$ ), and Pareto distributions (if $\alpha=1, \beta<0$ ).

If $a=0$, we have an extension of two-parameter Burr $\mathrm{X}$ distribution which is introduced by Surles and Padgett (2005) and also is known as generalized Rayleigh distribution (Kundu and Raqab, 2005). Therefore, it is called extended generalized Rayleigh (EGR) distribution.

Proposition 1. If $X$ has a EGLFR distribution with the pdf in (2.2), then $G(X)=a X+\frac{b}{2} X^{2}$ has a EGE distribution with parameters $\alpha, \beta$, and 1 or equivalently, $Y^{*}=X+\frac{b}{2 a} X^{2}$ has a EGE distribution with parameters $\alpha, \beta$, and $a$.

Proposition 2. Suppose that $X$ has the following $c d f$

$$
F_{1}(x)=\left\{\begin{array}{lll}
1-(1-\beta z)^{1 / \beta} & \text { if } & \beta \neq 0 \\
1-\mathrm{e}^{-z} & \text { if } & \beta=0
\end{array}\right.
$$

where $z=a x+\frac{b}{2} x^{2}$. We say that $X$ has extended linear failure rate (ELFR) distribution. The maximum of a random sample with size $n$ from the $\operatorname{ELFR}$ distribution has $\operatorname{EGLFR}(n, \beta, a, b)$. Therefore, the $\operatorname{EGLFR}(n, \beta, a, b)$ provides the cdf of a parallel system when each component has the ELFR distribution.

Theorem 2.2. Let $f(x)$ be the pdf of the EGLFR distribution. The limiting behavior of $f(x)$ for different values of its parameters is given bellow:

$$
\lim _{x \rightarrow 0^{+}} f(x)=\left\{\begin{array}{ll}
0 & \text { if } \quad \alpha>1 \\
a & \text { if } \quad \alpha=1 \\
\infty & \text { if } \quad \alpha<1
\end{array} \text { and } \quad \lim _{x \rightarrow c^{-}} f(x)= \begin{cases}\infty & \text { if } \beta \geq 1 \\
0 & \text { if } \beta<1\end{cases}\right.
$$

where $c=\psi$ for $\beta>0$, and $c=\infty$ for $\beta<0$.

Proof. The proof is obvious.

Theorem 2.3. Let $f(x)$ be the pdf of the EGLFR distribution. Then,

$i$. the mode of $f(x)$ is obtained from the solution of the following nonlinear equation when $\alpha \geq 1, \beta<1$ and $\beta \neq 0$ :

$$
\frac{b}{a+b x}+\frac{(\beta-1)(a+b x)}{1-\beta\left(a x+\frac{b}{2} x^{2}\right)}+(\alpha-1) \frac{(a+b x)\left(1-\beta\left(a x+\frac{b}{2} x^{2}\right)\right)^{\frac{1}{\beta}-1}}{1-\left(1-\beta\left(a x+\frac{b}{2} x^{2}\right)\right)^{1 / \beta}}=0,
$$

ii. the mode of $f(x)$ is obtained from the solution of the following nonlinear equation when $\alpha \geq 1$ and $\beta=0$ :

$$
\frac{b}{a+b x}-(a+b x)+(\alpha-1) \frac{a+b x}{\mathrm{e}^{a x+\frac{b}{2} x^{2}}-1}=0
$$


iii. the mode of $f(x)$ is 0 when $\alpha<1$ and $\beta<1$,

iv. $f(x)$ has two modes at 0 and $\psi$ when $\alpha<1$ and $\beta \geq 1$.

Proof. Using Theorem 2.2, the proof is obvious.

Theorem 2.4. Let $F$ be the pdf of the EGLFR distribution. Then, $F$ is a heavy-tailed distribution when $\alpha \geq 1$ and $\beta<0$.

Proof. Consider $z=a x+\frac{b}{2} x^{2}$ and $s=-\frac{1}{\beta}$. Therefore,

$$
\bar{F}(x)=1-F(x)=1-\left(1-\left(1+\frac{z}{s}\right)^{-s}\right)^{\alpha} .
$$

Without loss of generality take $\alpha=1$. Clearly, we have $\bar{F}(x) \sim\left(\frac{s}{z}\right)^{s}$, as $x \rightarrow \infty$. So

$$
\lim _{x \rightarrow \infty}\left(\frac{s}{z}\right)^{s} \mathrm{e}^{\lambda x}=\infty \quad \text { for } \forall \lambda>0
$$

and $F$ is a heavy tailed distribution (see Foss et al., 2011, Theorem 2.6).

The hrf of the EGLFR distribution is

$$
h(x)=\left\{\begin{array}{lll}
\frac{\alpha(a+b x)(1-\beta z)^{\frac{1}{\beta}-1}\left(1-(1-\beta z)^{1 / \beta}\right)^{\alpha-1}}{1-\left(1-(1-\beta z)^{1 / \beta}\right)^{\alpha}} & \text { if } & \beta \neq 0 \\
\frac{\alpha(a+b x) \mathrm{e}^{-z}\left(1-\mathrm{e}^{-z}\right)^{\alpha-1}}{1-\left(1-\mathrm{e}^{-z}\right)^{\alpha}} & \text { if } & \beta=0,
\end{array}\right.
$$

where $z=a x+\frac{b}{2} x^{2}$. For some cases of parameters, the plot for hazard of EGLFR distribution are given in Figure 2 .
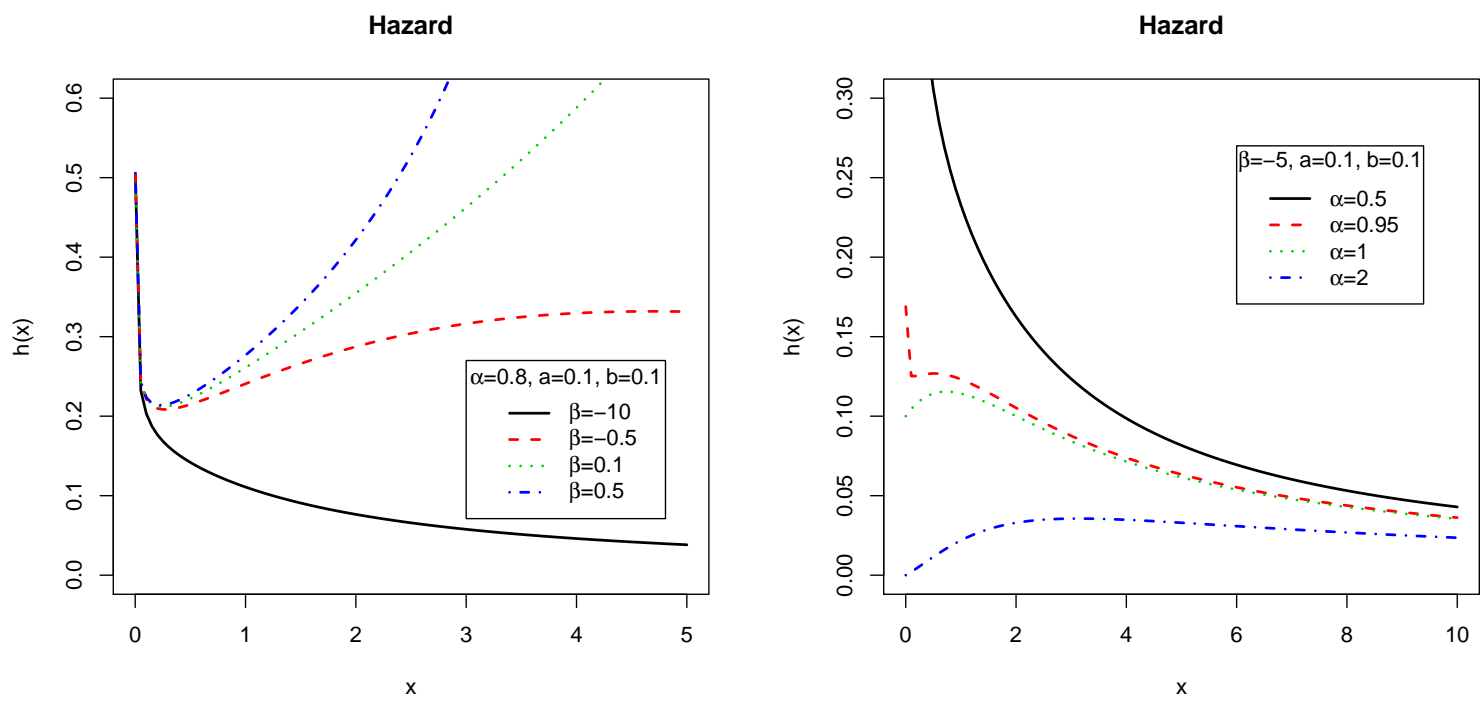

Figure 2: The plot of hrf for some different values of parameters. 
Theorem 2.5. Let $h(x)$ be the hrf of the EGLFR distribution. The limiting behavior of $h(x)$ for different values of its parameters is given bellow:

$$
\lim _{x \rightarrow 0^{+}} h(x)=\left\{\begin{array}{ll}
0 & \text { if } \quad \alpha>1 \\
a & \text { if } \quad \alpha=1 \\
\infty & \text { if } \quad \alpha<1
\end{array} \quad \text { and } \quad \lim _{x \rightarrow c^{-}} h(x)= \begin{cases}\infty & \text { if } \beta>0 \\
0 & \text { if } \beta<0\end{cases}\right.
$$

where $c=\psi$ for $\beta>0$, and $c=\infty$ for $\beta<0$.

Proof. The proof is obvious.

Theorem 2.6. Let $h(x)$ be the hrf of the EGLFR distribution. Then $h(x)$ is increasing function for $\beta \geq 1$ and $\alpha \geq 1$,

Proof. For $b=0$, we have the EGE distribution and proof is given by Kundu and Gupta (2011).

Here, we consider $b>0$. Let $z=a x+\frac{b}{2} x^{2}=\frac{b}{2}\left(x+\frac{a}{b}\right)^{2}-\frac{a^{2}}{2 b}$. Then, $x=\frac{1}{b} \sqrt{2 b z+a^{2}}-\frac{a}{b}$, and for $\beta \neq 0$

$$
h(z)=\frac{\alpha \sqrt{2 b z+a^{2}}(1-\beta z)^{\frac{1}{\beta}-1}\left(1-(1-\beta z)^{1 / \beta}\right)^{\alpha-1}}{1-\left(1-(1-\beta z)^{1 / \beta}\right)^{\alpha}} .
$$

With $u(z)=\log (h(z))$, we have

$$
\begin{aligned}
\frac{\partial}{\partial z} u(z)= & \frac{b}{2 b z+a^{2}}+(\beta-1) \frac{1}{1-\beta z}+(\alpha-1) \frac{(1-\beta z)^{\frac{1}{\beta}-1}}{1-(1-\beta z)^{\frac{1}{\beta}}} \\
& +\frac{\alpha}{\beta} \frac{\left(1-(1-\beta z)^{1 / \beta}\right)^{\alpha-1}(1-\beta z)^{1 / \beta-1}}{1-\left(1-(1-\beta z)^{1 / \beta}\right)^{\alpha}} .
\end{aligned}
$$

If $\beta \geq 1$ and $\alpha \geq 1$, then the hrf is an increasing function.

The reversible hazard function of EGLFR distribution is

$$
r(x ; \alpha, \beta, a, b)=\frac{f(x ; \alpha, \beta, a, b)}{F(x ; \alpha, \beta, a, b)}=\frac{\alpha(a+b x)\left(1-\beta\left(a x+\frac{b}{2} x^{2}\right)\right)^{\frac{1}{\beta}-1}}{\left(1-\left(1-\beta\left(a x+\frac{b}{2} x^{2}\right)\right)^{1 / \beta}\right)} .
$$

It is clear that $r(x ; \alpha, \beta, a, b)=\alpha r(x ; 1, \beta, a, b)$.

\section{Properties of EGLFR distribution}

We provide some of statistical properties of introduced distribution such as moments and quantiles. For more properties, reader can see Sarhan and Kundu (2009) and Kundu and Gupta (2011) for $\beta=0$ and $b=0$, respectively. 


\subsection{Quantiles of EGLFR distribution}

The quantile function of EGLFR distribution is

$$
Q(u)=\left\{\begin{array}{lll}
\frac{1}{b} \sqrt{a^{2}+\frac{2 b}{\beta}\left[1-(1-\sqrt[\alpha]{u})^{\beta}\right]}-\frac{a}{b} & \text { if } \beta \neq 0, b>0 \\
\frac{1}{b} \sqrt{a^{2}-2 b \log (1-\sqrt[\alpha]{u})}-\frac{a}{b} & \text { if } \beta=0, b>0 \\
\frac{1}{\beta a}\left(1-(1-\sqrt[\alpha]{u})^{\beta}\right) & \text { if } \beta \neq 0, b=0 \\
-\frac{1}{a} \log (1-\sqrt[\alpha]{u}) & \text { if } \beta=0, b=0 .
\end{array}\right.
$$

The median of the EGLFR distribution can be obtain by letting $u=0.5$. We can use this function for generating data form EGLFR distribution by generating data from a uniform distribution. For checking, we have generated a random sample from $\operatorname{EGLFR}(0.8,2,0.5,0.1)$. The histogram of this random sample and pdf of EGLFR are plotted in Figure 3 (left). In addition, the empirical cdf of this sample and cdf of EGLFR are plotted in Figure 3 (right).
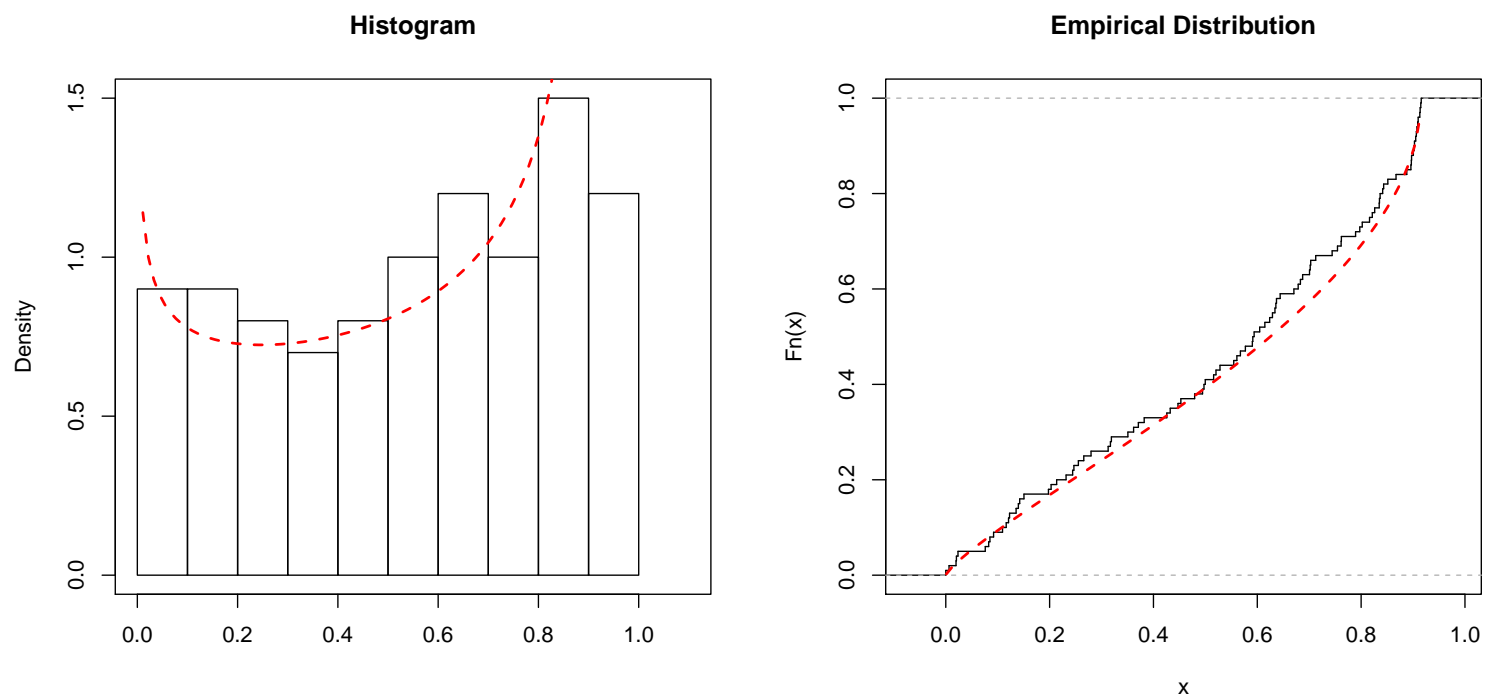

Figure 3: The histogram of the simulated data set and the pdf of EGLFR distribution (left); the empirical cdf of the simulated data set and the cdf of EGLFR distribution (right).

\subsection{Skewness and kurtosis of EGLFR distribution}

The Bowley skewness (see Kenney and Keeping, 1962) (based on quantiles can be calculated by

and the Moors kurtosis (see Moors, 1988) is defined as

$$
B=\frac{Q\left(\frac{3}{4}\right)-2 Q\left(\frac{1}{2}\right)+Q\left(\frac{1}{4}\right)}{Q\left(\frac{3}{4}\right)-Q\left(\frac{1}{4}\right)},
$$

$$
M=\frac{Q\left(\frac{7}{8}\right)-Q\left(\frac{5}{8}\right)+Q\left(\frac{3}{8}\right)-Q\left(\frac{1}{8}\right)}{Q\left(\frac{6}{8}\right)-Q\left(\frac{2}{8}\right)} .
$$


These measures are less sensitive to outliers and they exist even for distributions without moments. For the standard normal and the classical standard $t$ distributions with 10 degrees of freedom, the Bowley measure is 0. The Moors measure for these distributions is 1.2331 and 1.27705, respectively. In Figure 4, we plot Bowley and Moors measures (with $a=b=0.1$ ) as a function of $\beta$ for fixed values of $\alpha$.
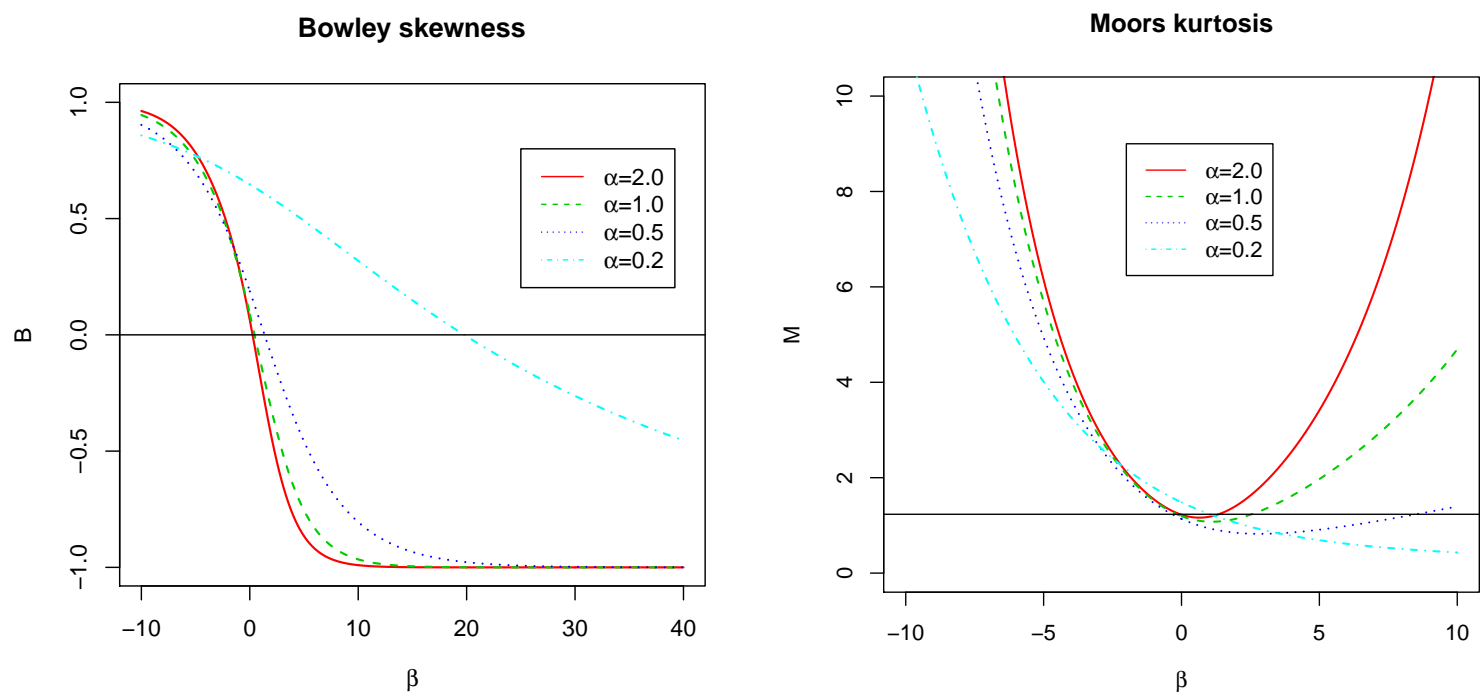

Figure 4: Plot of Bowley measure (left) and Moors measure for some parameters. (right).

\subsection{Moments of EGLFR distribution}

Using the following theorem, we can calculate the $r$-th non-central moment, $\mu^{(r)}$, of EGLFR distribution in a series form.

Theorem 3.1. Let $X$ has $a \operatorname{EGLFR}(\alpha, \beta, a, b)$. Then, for $\beta>0$ and $b>0$,

$$
\mu^{(r)}=E\left(X^{r}\right)=\sum_{n=0}^{\infty} \sum_{m=0}^{\infty} \sum_{k=0}^{m} \frac{\alpha}{2^{k}}(-1)^{m+n} \Lambda_{[n, m, k]} \beta^{m} a^{m-k} b^{k} \psi^{j}\left(\frac{a}{j}+\frac{b \psi}{j+1}\right)
$$

where $j=m+k+r+1$ and $\Lambda_{[n, m, k]}=\left(\begin{array}{c}\alpha-1 \\ n\end{array}\right)\left(\begin{array}{c}\frac{n+1}{\beta}-1 \\ m\end{array}\right)\left(\begin{array}{c}m \\ k\end{array}\right)$.

Proof. Consider $z=a x+\frac{b}{2} x^{2}$. If $\beta>0$, then support of $X$ is $(0, \psi)$. So, $\beta z<1$ and $(1-\beta z)^{1 / \beta}<1$. By using binomial series expansion, we have

$$
\begin{aligned}
& \left(1-(1-\beta z)^{1 / \beta}\right)^{\alpha-1}=\sum_{n=0}^{\infty}(-1)^{n}\left(\begin{array}{c}
\alpha-1 \\
n
\end{array}\right)(1-\beta z)^{\frac{n}{\beta}} \\
& (1-\beta z)^{\frac{n+1}{\beta}-1}=\sum_{m=0}^{\infty}(-1)^{m}\left(\begin{array}{c}
\frac{n+1}{\beta}-1 \\
m
\end{array}\right)(\beta z)^{m},
\end{aligned}
$$




$$
\left(a+\frac{b}{2} x\right)^{m}=\sum_{k=0}^{m}\left(\begin{array}{c}
m \\
k
\end{array}\right) a^{m-k} \frac{b^{k}}{2^{k}} x^{k} .
$$

Therefore,

$$
\begin{aligned}
& \mu^{(r)}=\int_{0}^{\psi} x^{r} \alpha(a+b x)(1-\beta z)^{\frac{1}{\beta}-1}\left(1-(1-\beta z)^{1 / \beta}\right)^{\alpha-1} d x \\
& =\int_{0}^{\psi} \alpha x^{r}(a+b x) \sum_{n=0}^{\infty}(-1)^{n}\left(\begin{array}{c}
\alpha-1 \\
n
\end{array}\right)(1-\beta z)^{\frac{n+1}{\beta}-1} d x \\
& =\int_{0}^{\psi} \alpha x^{r}(a+b x) \sum_{n=0}^{\infty} \sum_{m=0}^{\infty}(-1)^{n+m}\left(\begin{array}{c}
\alpha-1 \\
n
\end{array}\right)\left(\begin{array}{c}
\frac{n+1}{\beta}-1 \\
m
\end{array}\right) \beta^{m}\left(a+\frac{b}{2} x\right)^{m} d x \\
& =\int_{0}^{\psi} \alpha x^{r}(a+b x) \sum_{n=0}^{\infty} \sum_{m=0}^{\infty} \sum_{k=0}^{m}(-1)^{n+m}\left(\begin{array}{c}
\alpha-1 \\
n
\end{array}\right)\left(\begin{array}{c}
\frac{n+1}{\beta}-1 \\
m
\end{array}\right)\left(\begin{array}{c}
m \\
k
\end{array}\right) \beta^{m} a^{m-k} \frac{b^{k}}{2^{k}} x^{m+k} d x \\
& =\int_{0}^{\psi} \alpha x^{r}(a+b x) \sum_{n=0}^{\infty} \sum_{m=0}^{\infty} \sum_{k=0}^{m}(-1)^{n+m} \Lambda_{[n, m, k]} \beta^{m} a^{m-k} \frac{b^{k}}{2^{k}} x^{m+k} d x \\
& =\sum_{n=0}^{\infty} \sum_{m=0}^{\infty} \sum_{k=0}^{m} \frac{\alpha}{2^{k}}(-1)^{m+n} \Lambda_{[n, m, k]} \beta^{m} a^{m-k} b^{k} \int_{0}^{\psi} x^{m+k+r}(a+b x) d x \\
& =\sum_{n=0}^{\infty} \sum_{m=0}^{\infty} \sum_{k=0}^{m} \frac{\alpha}{2^{k}}(-1)^{m+n} \Lambda_{[n, m, k]} \beta^{m} a^{m-k} b^{k} \psi^{j}\left(\frac{a}{j}+\frac{b \psi}{j+1}\right),
\end{aligned}
$$

and proof is completed.

Theorem 3.2. Let $X$ has a $\operatorname{EGLFR}(\alpha, \beta, a, b)$. Then, for $\beta>0$ and $b>0$,

$$
\begin{aligned}
& M_{X}(t)=E\left(\mathrm{e}^{t x}\right)=\sum_{n=0}^{\infty} \sum_{m=0}^{\infty} \sum_{k=0}^{m} \sum_{i=0}^{m+k} \frac{(-1)^{n+m+i} \mathrm{e}^{t \psi}(m+k) !}{t^{i+1}(m+k-i) !} \frac{\alpha}{2^{k}} \Lambda_{[n, m, k]} \beta^{m} a^{m-k} b^{k} \psi^{m+k-i} \\
& \times\left[a+\frac{b(m+k+1) \psi}{(m+k+1-i)}\right]-\sum_{n=0}^{\infty} \sum_{m=0}^{\infty} \sum_{k=0}^{m} \frac{(-1)^{n+2 m+k}\left(a-\frac{b}{t}\right)}{t^{m+k+1}} \frac{\alpha}{2^{k}} \Lambda_{[n, m, k]} \beta^{m} a^{m-k} b^{k} .
\end{aligned}
$$

where $\Lambda_{[n, m, k]}=\left(\begin{array}{c}\alpha-1 \\ n\end{array}\right)\left(\begin{array}{c}\frac{n+1}{\beta}-1 \\ m\end{array}\right)\left(\begin{array}{c}m \\ k\end{array}\right)$.

Proof. Since (see Gradshteyn and Ryzhik, 2007, Section 2.321)

$$
\int x^{n} \mathrm{e}^{t x} d x=\mathrm{e}^{t x} n ! \sum_{i=0}^{n} \frac{(-1)^{i}}{t^{i+1}(n-i) !} x^{n-i}
$$

we have

$$
\begin{aligned}
\int_{0}^{\psi} x^{m+k}(a+b x) \mathrm{e}^{t x} d x & =a \int_{0}^{\psi} x^{m+k} \mathrm{e}^{t x} d x+b \int_{0}^{\psi} x^{m+k+1} \mathrm{e}^{t x} d x \\
& =\mathrm{e}^{t \psi}(m+k) ! \sum_{i=0}^{m+k} \frac{(-1)^{i}}{t^{i+1}(m+k-i) !} \psi^{m+k-i}\left[a+\frac{b(m+k+1) \psi}{(m+k+1-i)}\right]
\end{aligned}
$$




$$
-\frac{(-1)^{m+k}\left(a-\frac{b}{t}\right)}{t^{m+k+1}}
$$

Similar to Theorem 3.1, we have

$$
M_{X}(t)=\sum_{n=0}^{\infty} \sum_{m=0}^{\infty} \sum_{k=0}^{m} \frac{\alpha}{2^{k}}(-1)^{n+m} \Lambda_{[n, m, k]} \beta^{m} a^{m-k} b^{k} \int_{0}^{\psi} x^{m+k}(a+b x) \mathrm{e}^{t x} d x .
$$

and proof is completed.

Corollary 3.1. For $\beta>0, b>0$ and $a=0$, from Theorem 3.1 we have

$$
\mu^{(r)}=\sum_{n=0}^{\infty} \sum_{m=0}^{\infty} \frac{\alpha b}{(2 m+r+1)}(-1)^{n+m}\left(\begin{array}{c}
\alpha-1 \\
n
\end{array}\right)\left(\begin{array}{c}
\frac{n+1}{\beta}-1 \\
m
\end{array}\right)\left(\frac{2}{b \beta}\right)^{\frac{r+1}{2}}
$$

Remark 3.1. Using the equation (6) of Kundu and Gupta (2011) and proposition 1 for $Y=$ $a X+\frac{b}{2} X^{2}$, we have

$$
E\left((1-\beta Y)^{k}\right)=\frac{\Gamma(\alpha+1) \Gamma(k \beta+1)}{\Gamma(\alpha+k \beta+1)}:=\mathrm{g}_{k}, \quad k \beta+1>0, \quad \beta \neq 0 .
$$

When $a=0$,

$$
E\left(X^{2 k}\right)=(-1)^{k}\left(\frac{2}{\beta b}\right)^{k}\left(g_{k}-1-\sum_{r=1}^{k-1}(-1)^{r}\left(\begin{array}{l}
k \\
r
\end{array}\right)\left(\frac{\beta b}{2}\right)^{r} E\left(X^{2 r}\right)\right), \quad k=2,3, \ldots .
$$

Therefore,

$$
E\left(X^{2}\right)=\frac{2}{b \beta}\left(1-\mathrm{g}_{1}\right), \quad E\left(X^{4}\right)=\frac{4}{b^{2} \beta^{2}}\left(\mathrm{~g}_{2}-2 \mathrm{~g}_{1}+1\right) .
$$

Theorem 3.3. Let $X$ has a $\operatorname{EGLFR}(\alpha, \beta, a, b)$. Then, for $\beta<0$ and $\alpha \geq 1$, the $k$-th moment of $X$ does not exist when $k \geq-\frac{2}{\beta}$.

Proof. Consider $\alpha=1$. Then, the survival function of $X$ is

$$
\bar{F}(x)=\left(1+\frac{z}{s}\right)^{-s}
$$

where $z=a x+\frac{b}{2} x^{2}$. Since $X$ is a positive random variable, so

$$
E\left[X^{k}\right]=\int_{0}^{\infty} P\left(X>x^{1 / k}\right) d x=\int_{0}^{\infty} \bar{F}\left(x^{1 / k}\right) d x=\int_{0}^{\infty} \frac{d x}{\left(1+\frac{a x^{1 / k}+\frac{b}{2} x^{2 / k}}{s}\right)^{s}}
$$

where $s=-\frac{1}{\beta}$. clearly, $\left(s+a x^{1 / k}+\frac{b}{2} x^{2 / k}\right) / s \sim \frac{b}{2 s} x^{2 / k}$, as $x \rightarrow \infty$. The integral $\int_{0}^{\infty} x^{-2 s / k} d x$ diverges when $k \geq 2 s$, and therefore, $E\left[X^{k}\right]$ does not exists.

Consider $F_{\alpha}(x)=\left(1-\left(1+\frac{z}{s}\right)^{-s}\right)^{\alpha}$. It can be easily seen that if $\alpha_{1}<\alpha_{2}$ then $F_{\alpha_{1}}(x)>$ $F_{\alpha_{2}}(x)$ and for the survival function $\bar{F}(x)$ we have $\bar{F}_{\alpha_{1}}(x)<\bar{F}_{\alpha_{2}}(x)$. So, (at least) the divergence condition $k \geq 2 s$, satisfies for $\forall \alpha \geq 1$. 
Table 1: Some moments of EGLFR distribution for $\beta<0$.

\begin{tabular}{|c|c|cccccc|}
\hline & & \multicolumn{7}{|c|}{$\beta$} \\
\hline$\alpha$ & $k$ & -1.90 & -1.00 & -0.75 & -0.50 & -0.25 & -0.10 \\
\hline 1 & 1 & 20.195 & 2.221 & 1.829 & 1.571 & 1.388 & 1.303 \\
& 2 & - & - & 8.000 & 4.000 & 2.667 & 2.222 \\
& 3 & - & - & - & 18.849 & 6.664 & 4.598 \\
& 4 & - & - & - & - & 21.333 & 11.111 \\
& 5 & - & - & - & - & 88.857 & 30.656 \\
\hline 2 & 1 & 38.886 & 3.332 & 2.617 & 2.159 & 1.846 & 1.702 \\
& 2 & - & - & 14.400 & 6.667 & 4.190 & 3.391 \\
& 3 & - & - & - & 35.342 & 11.610 & 7.732 \\
& 4 & - & - & - & - & 39.619 & 19.883 \\
& 5 & - & - & - & - & 171.467 & 57.127 \\
\hline
\end{tabular}

Based on Remark 3.1, if $k$-th moment of EGLFR distribution exists, we have an expression for it when $k$ is even. But, we did not find an expression for other cases. In Table 1, we calculated some moments of EGLFR distribution for $\beta<0$ when they exist with considering $a=0$ and $b=1$.

\subsection{Entropy}

The entropy of random variable is defined in terms of its probability distribution and can be shown to be a good measure of randomness or uncertainty. The Shannon's entropy of a continuous random variable $Y$ with pdf $f(y)$ is defined by Shannon (1948) as

$$
H_{S h}(f)=-E_{f}[\log f(Y)]=-\int_{0}^{\infty} f(y) \log f(y) d y
$$

Since the pdf of EGLFR distribution can be written as

$$
f(x)=\alpha f_{1}(x)\left[F_{1}(x)\right]^{\alpha-1}
$$

where $f_{1}$ and $F_{1}$ are pdf and cdf of ELFR distribution, respectively, given in Proposition 2, the Shannon entropy for EGLFR distribution can be expressed in the form

$$
\begin{aligned}
H_{S h}(f)= & =-\ln (\alpha)+\frac{\alpha-1}{\alpha}-E_{f}\left[\ln f_{1}(X)\right] \\
& =-\ln (\alpha)+\frac{\alpha-1}{\alpha}-E_{W}\left[\ln f_{1}\left(F_{1}^{-1}(W)\right)\right]
\end{aligned}
$$

where $W$ has the beta distribution with parameters $\alpha$ and 1. The last term in (3.2) follows immediately from the conditions in Lemma 1 of Zografos and Balakrishnan (2009). The Rényi 
entropy is defined by

$$
H_{\rho}(f)=\frac{1}{1-\rho} \log \left(\int_{-\infty}^{\infty}[f(y)]^{\rho} d y\right)
$$

where $\rho>0$ and $\rho \neq 1$. The Shannon entropy is derived from $\lim _{\rho \rightarrow 1} H_{\rho}(f)$. An explicit expression of Rényi entropy for EGLFR distribution is obtained as

$$
H_{\rho}(f)=\frac{-\rho}{\rho-1} \ln (\alpha)-\frac{1}{\rho-1} \ln B(\rho(\alpha-1)+1,1)-\frac{1}{\rho-1} \ln E_{T}\left[f_{1}^{\rho-1}\left(F_{1}^{-1}(T)\right)\right],
$$

where $T$ has the beta distribution with parameters $\rho(\alpha-1)+1$ and 1 .

\subsection{Characterization}

Using the following theorem, we can characterize the EGLFR distribution.

Theorem 3.4. The random variable $X$ follows $\operatorname{EGLFR}(\alpha, \beta, a, b)$ if and only if for all real $t>0$, and for all non-negative integer $n$

$$
\delta^{(n)}(t)=U^{n}(t)+\frac{n}{\alpha} \delta^{(n-1)}(t)
$$

where $\delta^{(n)}(t)=E\left(U^{n}(X) \mid X<t\right)$ and $U(t)=-\log (\operatorname{red} D(t))=-\log \left(1-\left(1-\beta\left(a t+\frac{b}{2} t^{2}\right)\right)^{1 / \beta}\right)$.

Proof. Necessity condition:

Let $F$ and $f$ be the cdf and pdf of $\operatorname{EGLFR}(\alpha, \beta, a, b)$, respectively. Also, let $d(x)$ be the derivative function of $D(x)$. It can be shown that

$$
\begin{aligned}
\delta^{(n)}(t) & =\frac{\alpha}{F(t)} \int_{0}^{t}[-\log (D(x))]^{n} d(x)[D(x)]^{\alpha-1} d x \\
& =\frac{\alpha}{F(t)} \int_{0}^{D(t)}[-\log (z)]^{n} z^{\alpha-1} d z \\
& =\frac{1}{F(t)} \int_{0}^{D(t)}[-\log (z)]^{n} d z^{\alpha} \\
& =\left.\frac{[-\log (z)]^{n} z^{\alpha}}{F(t)}\right|_{0} ^{D(t)}+\frac{n}{F(t)} \int_{0}^{D(t)}[-\log (z)]^{n-1} z^{\alpha-1} d z \\
& =U^{n}(t)+\frac{n}{\alpha} \delta^{(n-1)}(t) .
\end{aligned}
$$

Sufficiency condition: Sarhan and Kundu (2009) showed that $\delta^{(n)}(t)=U^{n}(t)+\frac{n}{\alpha} \delta^{(n-1)}(t)$, implies that

$$
r(x)=\frac{f(x)}{F(x)}=\alpha \frac{f(x ; 1, \beta, a, b)}{F(x ; 1, \beta, a, b)}=\alpha r(x ; 1, \beta, a, b) .
$$

Therefore, from the uniqueness property of reversible hazard function, we can conclude that $X$ follows a $\operatorname{EGLFR}(\alpha, \beta, a, b)$. 


\section{Estimation}

In this section, we discuss the MLE of the parameters of the model when $\beta \neq 0$. Consider $X_{1}, \ldots, X_{n}$ is a random sample from EGLFR distribution with vector parameter $\boldsymbol{\theta}=$ $(\alpha, \beta, a, b)^{\prime}$. The log-likelihood function based on this random sample is given as

$\ell(\boldsymbol{\theta})=n \log (\alpha)+\sum_{i=1}^{n} \log \left(a+b x_{i}\right)+\left(\frac{1}{\beta}-1\right) \sum_{i=1}^{n} \log \left(1-\beta z_{i}\right)+(\alpha-1) \sum_{i=1}^{n} \log \left(1-\left(1-\beta z_{i}\right)^{\frac{1}{\beta}}\right)$,

where $z_{i}=a x_{i}+\frac{b}{2} x_{i}^{2}$. The log-likelihood can be maximized either directly or by solving the nonlinear likelihood equations obtained by differentiating (4.1). The components of the score vector $U(\boldsymbol{\theta})=\left(U_{\alpha}(\boldsymbol{\theta}), U_{\beta}(\boldsymbol{\theta}), U_{a}(\boldsymbol{\theta}), U_{b}(\boldsymbol{\theta})\right)^{T}$ are given by

$$
\begin{aligned}
U_{\alpha}(\boldsymbol{\theta})= & \frac{\partial \ell(\boldsymbol{\theta})}{\partial \alpha}=\frac{n}{\alpha}+\sum_{i=1}^{n} \log \left(1-\left(1-\beta z_{i}\right)^{\frac{1}{\beta}}\right), \\
U_{\beta}(\boldsymbol{\theta})= & \frac{\partial \ell(\boldsymbol{\theta})}{\partial \beta}=-\frac{1}{\beta^{2}} \sum_{i=1}^{n} \log \left(1-\beta z_{i}\right)-\left(\frac{1}{\beta}-1\right) \sum_{i=1}^{n} \frac{z_{i}}{1-\beta z_{i}} \\
& +\frac{\alpha-1}{\beta^{2}} \sum_{i=1}^{n} \frac{\left(1-\beta z_{i}\right)^{\frac{1}{\beta}-1}}{1-\left(1-\beta z_{i}\right)^{\frac{1}{\beta}}}\left[\beta z_{i}+\left(1-\beta z_{i}\right) \log \left(1-\beta z_{i}\right)\right], \\
U_{a}(\boldsymbol{\theta})= & \frac{\partial \ell(\boldsymbol{\theta})}{\partial a}=\sum_{i=1}^{n} \frac{1}{a+b x_{i}}-\left(\frac{1}{\beta}-1\right) \sum_{i=1}^{n} \frac{\beta x_{i}}{1-\beta z_{i}}+(\alpha-1) \sum_{i=1}^{n} \frac{x_{i}\left(1-\beta z_{i}\right)^{\frac{1}{\beta}-1}}{1-\left(1-\beta z_{i}\right)^{\frac{1}{\beta}}}, \\
U_{b}(\boldsymbol{\theta})= & \frac{\partial \ell(\boldsymbol{\theta})}{\partial b}=\sum_{i=1}^{n} \frac{x_{i}}{a+b x_{i}}-\left(\frac{1}{\beta}-1\right) \sum_{i=1}^{n} \frac{\beta x_{i}^{2}}{2\left(1-\beta z_{i}\right)}+(\alpha-1) \sum_{i=1}^{n} \frac{x_{i}^{2}\left(1-\beta z_{i}\right)^{\frac{1}{\beta}-1}}{2\left(1-\left(1-\beta z_{i}\right)^{\frac{1}{\beta}}\right)} .
\end{aligned}
$$

For given $\beta, a$, and $b$, the MLE of parameter $\alpha$ is

$$
\hat{\alpha}=-\frac{n}{\sum_{i=1}^{n} \log \left(1-\left(1-\beta z_{i}\right)^{\frac{1}{\beta}}\right)} .
$$

By maximizing the profile $\log$-likelihood $\ell(\hat{\alpha}, \beta, a, b)$ with respect to $\beta, a, b$, the MLE of these parameters can be obtained.

\subsection{The regular case}

When $\beta \leq 0$, the support of the EGLFR distribution is in interval $(0, \infty)$, and in this case the asymptotic distribution of the MLE of vector parameter $\boldsymbol{\theta}=(\alpha, \beta, a, b)^{\prime}$ is multivariate normal distribution as

$$
\sqrt{n}(\hat{\boldsymbol{\theta}}-\boldsymbol{\theta}) \stackrel{d}{\longrightarrow} N_{4}\left(\mathbf{0}, I^{-1}\right),
$$

where $I$ is the Fisher information matrix. 


\subsection{The non-regular case}

When $\beta>0$, the support of the EGLFR distribution is in interval $(0, \psi)$. So, in this case the standard asymptotic normality distribution of the MLE's of parameters does not hold. The asymptotic distributions are proposed by Kundu and Gupta (2011) for $b=0$. Here, similar results are given when $b>0$. The general approach is given by Smith (1985), and is used in literature (for example $\mathrm{Ng}$ et al., 2012).

Take $G(x)=a x+\frac{b}{2} x^{2}$. Then $G^{-1}\left(\frac{1}{\beta}\right)=\psi$, and the pdf of EGLFR distribution can be written as

$$
f(x ; \alpha, a, b, \psi)=\alpha \mathrm{g}(x)(1-t)^{G(\psi)-1}\left(1-(1-t)^{G(\psi)}\right)^{\alpha-1}, \quad x \leq \psi,
$$

where $t=G(x) / G(\psi)$, andg $(x)$ is the derivative of $G(x)$ with respect to $x$. So, the $\log$ likelihood function correspond to the pdf in (4.2) is

$$
\begin{aligned}
\ell(\alpha, a, b, \psi)= & n \log (\alpha)+\sum_{i=1}^{n} \log \left(\mathrm{g}\left(x_{(i)}\right)\right)+(G(\psi)-1) \sum_{i=1}^{n} \log \left(1-t_{(i)}\right) \\
& +(\alpha-1) \sum_{i=1}^{n} \log \left(1-\left(1-t_{(i)}\right)^{G(\psi)}\right)
\end{aligned}
$$

where $t_{(i)}=G\left(x_{(i)}\right) / G(\psi)$, and $x_{(1)}, x_{(2)}, \ldots, x_{(n)}$ are the ordered statistics of random sample $x_{1}, x_{2}, \ldots, x_{n}$. As mentioned in Smith (1985), at first, we find the MLE of the threshold parameter of the model. For the EGLFR distribution, the MLE of the threshold parameter $\psi$ is $\tilde{\psi}=x_{(n)}$. Then, the modified log-likelihood function based on the remaining $(n-1)$ observations is

$$
\begin{aligned}
\ell(\alpha, a, b, \tilde{\psi})= & (n-1) \log (\alpha)+\sum_{i=1}^{n-1} \log \left(\mathrm{g}\left(x_{(i)}\right)\right)+(G(\tilde{\psi})-1) \sum_{i=1}^{n-1} \log \left(1-\tilde{t}_{(i)}\right) \\
& +(\alpha-1) \sum_{i=1}^{n-1} \log \left(1-\left(1-\tilde{t}_{(i)}\right) G(\tilde{\psi})\right)
\end{aligned}
$$

where $\tilde{t}_{(i)}=G\left(x_{(i)}\right) / G(\tilde{\psi})$. For more information about the modified likelihood function, refer to Smith (1985). For fixed $a$ and $b$ the modified MLE of parameter $\alpha$ is obtained as

$$
\tilde{\alpha}=-\frac{n-1}{\sum_{i=1}^{n-1} \log \left(1-\left(1-\tilde{t}_{(i)}\right)^{G(\tilde{\psi})}\right)} .
$$

By maximizing the modified $\log$-likelihood $\ell(\tilde{\alpha}, a, b, \tilde{\psi})$ with respect to $a$, and $b$, the modified MLE of these parameters can be obtained. 
Theorem 4.1. The asymptotic distribution of $\tilde{\psi}$ is

$$
n^{1 / G(\psi)}(\tilde{\psi}-\psi) \stackrel{d}{\longrightarrow}-\frac{G(\psi)}{(a+b \psi)} V^{1 / G(\psi)},
$$

where the random variable $V$ is distributed as exponential distribution with mean $\frac{1}{\alpha}$.

Proof. The corresponding cdf of the pdf in (4.2) is

$$
F(x)=\left(1-(1-t)^{G(\psi)}\right)^{\alpha}
$$

So, $G(X) / G(\psi)$ is distributed as $1-\left(1-U^{1 / \alpha}\right)^{1 / G(\psi)}$ where $U$ has a standard uniform distribution. Therefore, $n^{1 / G(\psi)}\left(\frac{G\left(X_{(n)}\right)}{G(\psi)}-1\right)$ is distributed as $-n^{1 / G(\psi)}\left(1-U_{(n)}^{1 / \alpha}\right)^{1 / G(\psi)}$ where $U_{(n)}$ has a beta distribution with parameters $n$ and 1 . Now, we have

$$
\lim _{n \rightarrow \infty} P\left(n\left(1-U_{(n)}^{1 / \alpha}\right) \leq x\right)=1-\mathrm{e}^{-\alpha x}
$$

and then $n^{1 / G(\psi)}\left(G\left(X_{(n)}\right)-G(\psi)\right) \stackrel{d}{\longrightarrow}-G(\psi) V^{1 / G(\psi)}$. By using the delta method and the relation between the derivatives of the $G(x)$ and $G^{-1}(x)$, the proof is completed.

Theorem 4.2. a) Conditioning on $X_{(n)}$, the asymptotic distribution of the modified $M L E$, $(\tilde{\alpha}, \tilde{a}, \tilde{b})$ is multivariate normal distribution.

b) The asymptotic distribution of $(\tilde{\alpha}, \tilde{a}, \tilde{b})$ is (i) multivariate normal if $G(\psi)<\frac{1}{2}$, (ii) multivariate Weibull if $G(\psi)>\frac{1}{2}$, and (iii) a mixture of normal and Weibull if $G(\psi)=\frac{1}{2}$.

Proof. For the proof of this theorem, reader can be refer to Kundu and Gupta (2011).

\section{A real example}

The following data set is given by Aarset (1987) and represents the lifetimes of 50 devices. Sarhan and Kundu (2009) and Silva et al. (2010) also analyzed this data.

\subsection{0 .211111236711121818181818213236404546475055}

6063636767676772757982828384848485858585858686

Using this data, we obtained the MLE's of parameters of six distributions: extended generalized linear failure rate (EGLFR), extended generalized exponential (EGE), extended generalized Rayleigh (EGR), generalized linear failure rate (GLFR), generalized exponential (GE), and generalized Rayleigh (GR) distributions. The results are given in Table 2,

Based on the MLE's of parameters, we calculated minus of log-likelihood function $(-\log (L))$, Kolmogorov-Smirnov (K-S) statistic with its p-value, Akaike information criterion 
(AIC), Akaike information criterion corrected (AICC), Bayesian information criterion (BIC) and likelihood ratio test (LRT) with its p-value. Table 2 indicates that the GLFR and GE models are not suitable for this data set based on K-S statistic. Also, The EGLFR model has the lowest $-\log (L)$, AIC, AICC and BIC values among all fitted models. A comparison of the proposed distribution with some of its sub-models using p-value of LRT shows that the EGLFR model yields a better fit than the other five distributions to this real data set.

The beta modified Weibull (BMW) distribution was introduced by Silva et al. (2010) and has the following pdf:

$$
f_{B M W}(x)=\frac{\alpha x^{\gamma}(\gamma+\beta x)}{B(a, b)} \mathrm{e}^{\beta x-b \alpha x^{\gamma} \mathrm{e}^{\beta x}}\left(1-\mathrm{e}^{-\alpha x^{\gamma} \mathrm{e}^{\beta x}}\right)^{a-1}, \quad x>0,
$$

where $B(a, b)$ is the beta function with $\alpha, \beta, \gamma>0$ and $0<a, b<1$. This distribution contains several important distributions such as Weibull, modified Weibull (Lai et al., 2003), generalized exponential (Gupta and Kundu, 1999), beta Weibull (Lee et al.,, 2007) and generalized modified Weibull (Carrasco et al., 2008) distributions. Silva et al. (2010) showed that the BMW distribution produces a better fit than its sub-models for this data set. In Table 2, we present the MLE's, $-\log (L)$, and other criteria for the BMW distribution, and we can conclude that the EGLFR, EGR and EGE have better fit than the BMW distribution for this data set.

In Figure 5, we plot the histogram of this data set and the estimated pdf of the seven models. Moreover, the plots of empirical cdf of the data set and estimated cdf of the five models are displayed in Figure 5. Again, we can conclude that EGLFR distribution is a very satisfactory model for this data set.

There are various extensions of GLFR distribution in literature. The pdf of seven extensions of this distribution are given as follows. Here, we consider $\mathrm{g}(x)=(a+b x) \mathrm{e}^{-\left(a x+\frac{b}{2} x^{2}\right)}$ and $G(x)=1-\mathrm{e}^{-\left(a x+\frac{b}{2} x^{2}\right)}$, where $a>0$ and $b>0$.

\section{Beta LFR (BLFR) distribution}

This distribution is proposed by Jafari and Mahmoudi (2015) with the following pdf:

$$
f_{\mathrm{BLFR}}(x)=\frac{\mathrm{g}(x)}{B(\alpha, \beta)}(G(x))^{\alpha-1}(1-G(x))^{\beta-1}, \quad x>0,
$$

where $\alpha>0$ and $\beta>0$.

\section{Kumaraswamy GLFR (KGLFR) distribution}

This distribution is proposed by Elbatal (2013) with the following pdf:

$$
f_{\mathrm{KGLFR}}(x)=\alpha \beta \mathrm{g}(x)(G(x))^{\alpha-1}\left(1-(G(x))^{\alpha}\right)^{\beta-1}, \quad x>0,
$$


where $\alpha=c \theta$, and $\theta>0, c>0$ and $\beta>0$.

\section{McDonald GLFR (MCGLFR) distribution}

This distribution is proposed by Elbatal et al. (2014) with the following pdf:

$$
f_{\text {MCGLFR }}(x)=\frac{\gamma \mathrm{g}(x)}{B(\alpha, \beta)}(G(x))^{\alpha \gamma-1}\left(1-(G(x))^{\gamma}\right)^{\beta-1}, \quad x>0,
$$

where $\gamma=c \theta, \theta>0, c>0, \alpha>0$ and $\beta>0$.

\section{Modified GLFR (MGLFR) distribution}

This distribution is proposed by Jamkhaneh (2014) with the following pdf:

$$
f_{\text {MGLFR }}(x)=\alpha\left(a+b \beta x^{\beta-1}\right) e^{-\left(a x+b x^{\beta}\right)}\left(1-\mathrm{e}^{-\left(a x+b x^{\beta}\right)}\right)^{\alpha-1}, \quad x>0,
$$

where $a>0, b>0, \alpha>0$ and $\beta>0$.

\section{Poisson GLFR (PGLFR) distribution}

This distribution is proposed by Cordeiro et al. (2015) with the following pdf:

$$
f_{\mathrm{PGLFR}}(x)=\frac{\beta \alpha \mathrm{g}(x)}{\left(1-\mathrm{e}^{-\beta}\right)}(G(x))^{\alpha-1} \mathrm{e}^{-\beta(G(x))^{\alpha}}, \quad x>0,
$$

where $\alpha>0$ and $\beta>0$.

\section{Geometric GLFR (GGLFR) distribution}

This distribution is proposed by Nadarajah et al. (2014) with the following pdf:

$$
f_{\text {GGLFR }}(x)=\frac{\alpha(1-\beta) \mathrm{g}(x)(G(x))^{\alpha-1}}{\left(1-\beta\left(1-(G(x))^{\alpha}\right)\right)^{2}}, \quad x>0,
$$

where $\alpha>0$ and $\beta<1$.

\section{Generalized Linear Exponential (GLE) distribution}

This distribution is proposed by Mahmoud and Alam (2010) with the following pdf:

$$
f_{\mathrm{GGLFR}}(x)=\alpha(a+b x)\left(a x+\frac{b}{2} x^{2}-\beta\right)^{\alpha-1} \mathrm{e}^{-\left(a x+\frac{b}{2} x^{2}-\beta\right)^{\alpha}}, \quad x>0,
$$

where $a>0, b>0, \alpha>0$ and $\beta>0$.

We also fitted these distributions to the data set, and obtained the MLE's, $-\log (L)$ and other criteria for them. The results are given in Table 3. It can be concluded that the EGLFR, EGR and EGE have better fit than these seven extension of GLFR distribution for this data set. The histogram of the data set with the estimated pdf's and the empirical cdf of the data set with estimated cdf's for distributions are given in Figure 6. 
Table 2: MLE's of the model parameters, and the K-S, AIC, AICC, BIC, and LRT statistics.

\begin{tabular}{|l|c|c|c|c|c|c|c|}
\hline & \multicolumn{7}{|c|}{ Distribution } \\
\hline Statistic & EGLFR & EGE & EGR & GLFR & GE & GR & BMW \\
\hline$\hat{\alpha}$ & 0.2620 & 0.5368 & 0.2590 & 0.5327 & 0.7798 & 0.3520 & 0.0002 \\
$\hat{\beta}$ & 4.5000 & 1.8199 & 4.2100 & - & - & - & 0.0541 \\
$\hat{a}$ & $1.21 \times 10^{-8}$ & 0.0064 & - & 0.0038 & 0.0187 & - & 0.1975 \\
$\hat{b}$ & 0.00006 & - & 0.00006 & 0.0003 & - & 0.0003 & 0.1647 \\
$\hat{\gamma}$ & - & - & - & - & - & - & 1.3771 \\
\hline$-\log (L)$ & 173.9487 & 189.1973 & 180.5367 & 233.1447 & 239.9951 & 234.5655 & 220.6601 \\
K-S & 0.0981 & 0.1558 & 0.0872 & 0.1832 & 0.2042 & 0.2011 & 0.0846 \\
p-value (K-S) & 0.7215 & 0.1763 & 0.8413 & 0.0696 & 0.0309 & 0.0350 & 0.3971 \\
AIC & 355.8974 & 384.3945 & 367.0733 & 472.2895 & 483.9903 & 473.1309 & 451.3201 \\
AICC & 356.7863 & 384.9163 & 367.5951 & 472.8110 & 484.2456 & 473.3862 & 452.6838 \\
BIC & 363.5455 & 390.1306 & 372.8094 & 478.0256 & 487.8143 & 476.9550 & 460.8802 \\
LRT & - & 30.4971 & 13.1759 & 118.3921 & 132.0929 & 121.2335 & - \\
p-value (LRT) & - & 0.0000 & 0.0002 & 0.0000 & 0.0000 & 0.0000 & - \\
\hline
\end{tabular}
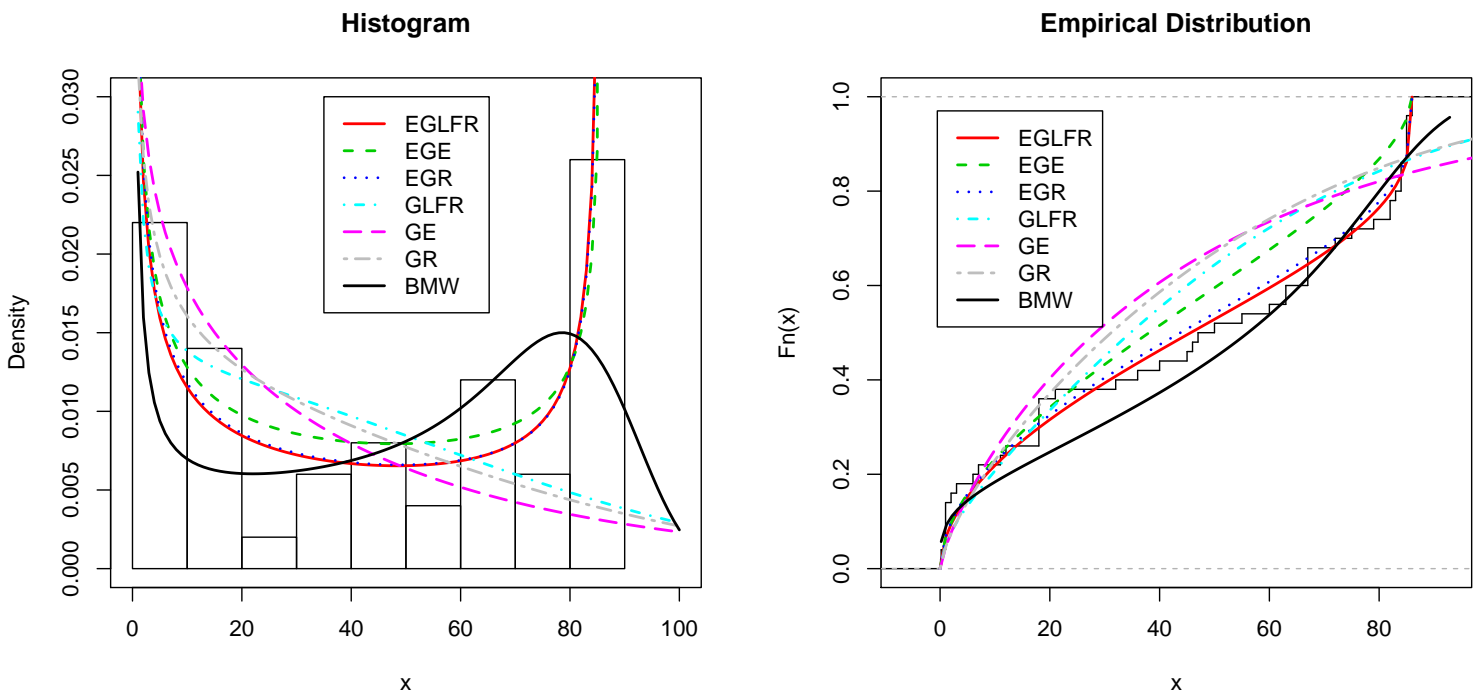

Figure 5: The histogram of the data set with the estimated pdf's (left); the empirical cdf of the data set and estimated cdf's (right). 
Table 3: MLEs of the model parameters, and the K-S (and its p-value), AIC, AICC, BIC for other extensions of GLFR distribution.

\begin{tabular}{|l|c|c|c|c|c|c|c|}
\hline & \multicolumn{7}{|c|}{ Distribution } \\
\hline Statistic & BLFR & KGLFR & MCGLFR & MGLFR & PGLFR & GGLFR & GLE \\
\hline$\hat{\alpha}$ & 0.3347 & 0.6525 & 0.0295 & 19699.45 & 0.5327 & 0.2624 & 0.6262 \\
$\hat{\beta}$ & 0.1243 & 0.0622 & $5.74 \times 10^{8}$ & 0.0164 & $10^{-8}$ & -5.5536 & 0.0015 \\
$\hat{a}$ & 0.0172 & 0.2988 & 0.0015 & 0.0246 & 0.0038 & 0.0086 & 0.0149 \\
$\hat{b}$ & 0.0035 & 0.0007 & $6.66 \times 10^{-5}$ & 8.8393 & 0.0003 & 0.0005 & 0.0005 \\
$\hat{\gamma}$ & - & - & 1.8936 & - & - & - & - \\
\hline$-\log (L)$ & 230.3785 & 238.0490 & 221.9929 & 235.3460 & 233.1447 & 229.9373 & 227.1663 \\
K-S & 0.1554 & 0.1666 & 0.1949 & 0.1624 & 0.1832 & 0.1297 & 0.2327 \\
p-value $($ K-S $)$ & 0.1784 & 0.1246 & 0.0448 & 0.1428 & 0.0696 & 0.3694 & 0.0088 \\
AIC & 468.7570 & 484.0980 & 453.9858 & 478.6921 & 474.2895 & 467.8745 & 462.3327 \\
AICC & 469.6459 & 484.9869 & 455.3494 & 479.5810 & 475.1784 & 468.7634 & 463.2216 \\
BIC & 476.4051 & 491.7461 & 463.5459 & 486.3402 & 481.9376 & 475.5226 & 469.9808 \\
\hline
\end{tabular}
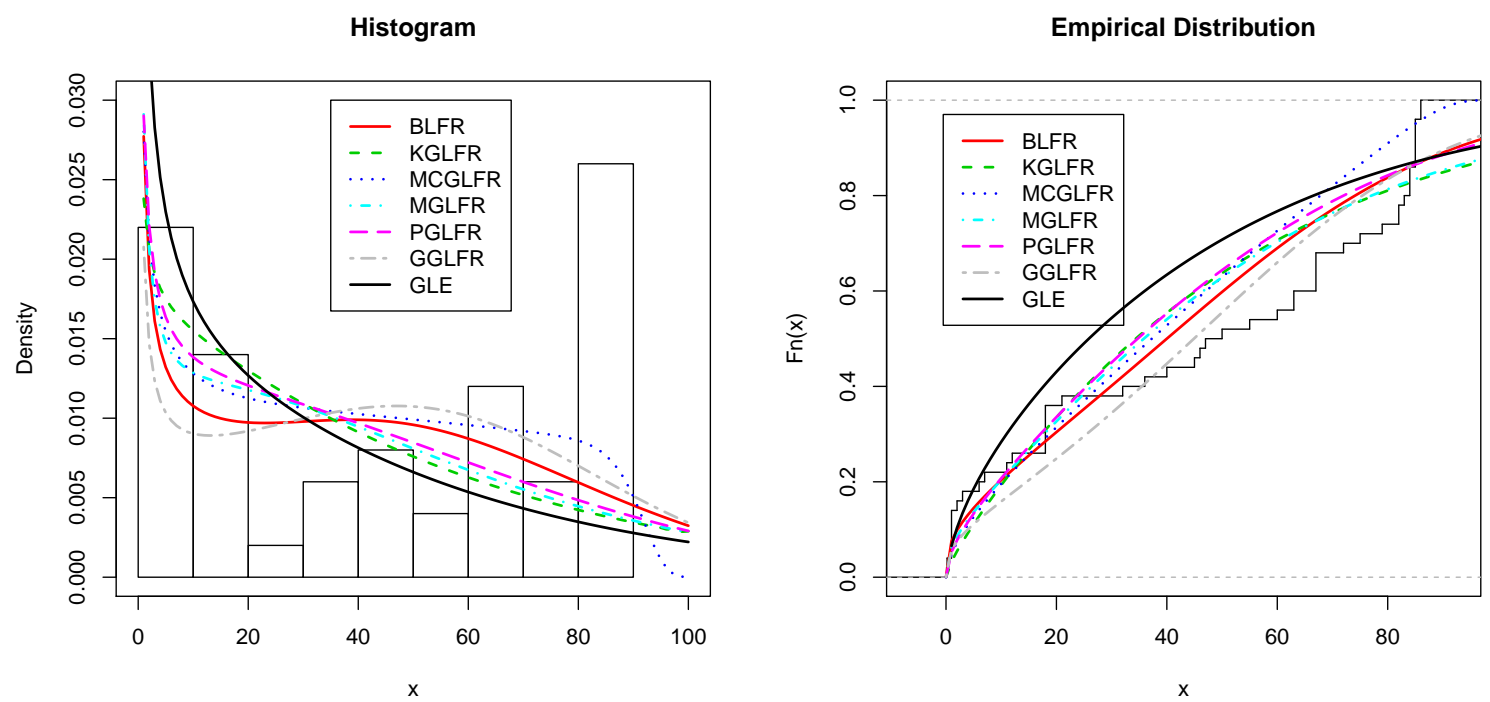

Figure 6: The histogram of the data set with the estimated pdf's (left); the empirical cdf of the data set and estimated cdf's (right) for seven extensions of GLFR distribution. 


\section{Conclusion}

In this paper, a four-parameter EGLFR distribution has been proposed. Although the GLFR distribution cannot have a unimodal hazard rate function, but EGLFR distribution can have a decreasing, increasing, unimodal, and bathtub-shaped hazard rate function depending on its parameters. This new class of distributions includes some well-known distribution such as EGE and GLFR distributions as special sub-models. Several properties of EGLFR distribution such as quantiles function, Skewness and kurtosis measures, and the $r$-th non-central moment have been given. the maximum likelihood estimators of the parameters are given and also, the asymptotic distribution of estimates are investigated. By using a real data set, we have shown that our new distribution fits to the lifetime data very well than the well-known distributions. Recently Sarhan et al. (2011) introduced the bivariate GLFR distribution and its multivariate extension. So, one can use the approach used in this paper to develop the multivariate EGLFR distribution.

\section{Acknowledgements}

The authors would like to thank the anonymous referees for many helpful comments and suggestions.

\section{References}

Aarset, M. V. (1987). How to identify a bathtub hazard rate. IEEE Transactions on Reliability, R-36(1):106-108.

Alamatsaz, M. H. and Shams, S. (2014). Generalized linear failure rate power series distribution. Communications in Statistics: Theory and Methods, In press.

Carrasco, J. M., Ortega, E. M., and Cordeiro, G. M. (2008). A generalized modified Weibull distribution for lifetime modeling. Computational Statistics $\&$ Data Analysis, 53(2):450-462.

Cordeiro, G. M., Hashimoto, E. M., Ortega, E. M., and Pascoa, M. A. (2012). The McDonald extended distribution: properties and applications. AStA Advances in Statistical Analysis, 96(3):409-433.

Cordeiro, G. M., Ortega, E. M. M., and Lemonte, A. J. (2015). The Poisson generalized linear failure rate model. Communications in Statistics-Theory and Methods, 44(10):2037-2058. 
Elbatal, I. (2013). Kumaraswamy generalized linear failure rate distribution. Indian Journal of Computational \& Applied Mathematics, 1(1):61-78.

Elbatal, I., Merovci, F., and Marzouk, W. (2014). McDonald generalized linear failure rate distribution. Pakistan Journal of Statistics and Operation Research, 10(3):267-288.

Foss, S., Korshunov, D., and Zachary, S. (2011). An Introduction to Heavy-Tailed and Subexponential Distributions. Springer, New York.

Gradshteyn, I. S. and Ryzhik, I. M. (2007). Table of Integrals, Series, and Products, Edited by Alan Jeffrey and Daniel Zwillinger. Academic Press, New York, 7th edition.

Gupta, R. D. and Kundu, D. (1999). Generalized exponential distributions. Australian \& New Zealand Journal of Statistics, 41(2):173-188.

Jafari, A. A. and Mahmoudi, E. (2015). Beta-linear failure rate distribution and its applications. Journal of the Iranian Statistical Society, Accepted for publication.

Jamkhaneh, E. B. (2014). Modified generalized linear failure rate distribution: Properties and reliability analysis. International Journal of Industrial Engineering Computations, 5(3):375386.

Johnson, N. L., Kotz, S., and Balakrishnan, N. (1995). Continuous Univariate Distributions, volume 2. John Wiley \& Sons, New York, second edition.

Kenney, J. F. and Keeping, E. (1962). Mathematics of Statistics. D. Van Nostrand Company.

Kundu, D. and Gupta, R. D. (2011). An extension of the generalized exponential distribution. Statistical Methodology, 8(6):485-496.

Kundu, D. and Raqab, M. Z. (2005). Generalized Rayleigh distribution: different methods of estimations. Computational Statistics \& Data Analysis, 49(1):187-200.

Lai, C., Xie, M., and Murthy, D. (2003). A modified Weibull distribution. IEEE Transactions on Reliability, 52(1):33-37.

Lee, C., Famoye, F., and Olumolade, O. (2007). Beta-Weibull distribution: some properties and applications to censored data. Journal of Modern Applied Statistical Methods, 6(1):173-186.

Mahmoud, M. A. W. and Alam, F. M. A. (2010). The generalized linear exponential distribution. Statistics \& Probability Letters, 80(1112):1005-1014. 
Mahmoudi, E. and Jafari, A. A. (2015). The compound class of linear failure rate-power series distributions: model, properties and applications. Communications in Statistics - Simulation and Computation, 10.1080/03610918.2015.1005232.

Moors, J. J. A. (1988). A quantile alternative for kurtosis. Journal of the Royal Statistical Society. Series D (The Statistician), 37(1):25-32.

Nadarajah, S., Shahsanaei, F., and Rezaei, S. (2014). A new four-parameter lifetime distribution. Journal of Statistical Computation and Simulation, 84(2):248-263.

Ng, H. K. T., Luo, L., Hu, Y., and Duan, F. (2012). Parameter estimation of three-parameter Weibull distribution based on progressively type-II censored samples. Journal of Statistical Computation and Simulation, 82(11):1661-1678.

Sarhan, A. M., Hamilton, D. C., Smith, B., and Kundu, D. (2011). The bivariate generalized linear failure rate distribution and its multivariate extension. Computational Statistics and Data Analysis, 55(1):644-654.

Sarhan, A. M. and Kundu, D. (2009). Generalized linear failure rate distribution. Communications in Statistics-Theory and Methods, 38(5):642-660.

Shannon, C. (1948). A mathematical theory of communication. Bell System Technical Journal, $27: 379-432$.

Silva, G. O., Ortega, E. M., and Cordeiro, G. M. (2010). The beta modified Weibull distribution. Lifetime Data Analysis, 16(3):409-430.

Smith, R. L. (1985). Maximum likelihood estimation in a class of nonregular cases. Biometrika, $72(1): 67-90$.

Surles, J. and Padgett, W. (2005). Some properties of a scaled Burr type X distribution. Journal of Statistical Planning and Inference, 128(1):271-280.

Surles, J. G. and Padgett, W. J. (2001). Inference for reliability and stress-strength for a scaled Burr type X distribution. Lifetime Data Analysis, 7(2):187-200.

Zografos, K. and Balakrishnan, N. (2009). On families of beta-and generalized gammagenerated distributions and associated inference. Statistical Methodology, 6(4):344-362. 